\title{
Melittin Adsorption and Lipid Monolayer Disruption at Liquid-Liquid Interfaces
}

\author{
Manuel A. Méndez, ${ }^{+, \|}$Zahra Nazemi, ${ }^{+, \neq, \|}$Ibrahim Uyanik, ${ }^{\S} \mathrm{Yu} \mathrm{Lu}{ }^{\dagger}$ and Hubert H. Girault ${ }^{*,+}$ \\ ${ }^{\dagger}$ Laboratoire d'Electrochimie Physique et Analytique, Ecole Polytechnique Fédérale de Lausanne (EPFL), Station 6, \\ CH-1015 Lausanne, Switzerland \\ ${ }^{\ddagger}$ Chemistry Department, University of Isfahan, Isfahan 81746-73441, Iran \\ ${ }^{\S}$ Department of Chemistry, Faculty of Science, Selcuk University, Campus, 42075 Konya, Turkey \\ S Supporting Information
}

ABSTRACT: Melittin, a membrane-active peptide with antimicrobial activity, was investigated at the interface formed between two immiscible electrolyte solutions (ITIES) supported on a metallic electrode. Ion-transfer voltammetry showed well-defined semi-reversible transfer peaks along with adsorptive peaks. The reversible adsorption of melittin at the liquid-liquid interface is qualitatively discussed from voltammetric data and experimentally confirmed by real-time image analysis of video snapshots. It is also demonstrated that polarization of the water/1,2-DCE interface results in drastic drop shape variations caused by large variations of the interfacial tension. The experimental data also confirmed that maximum

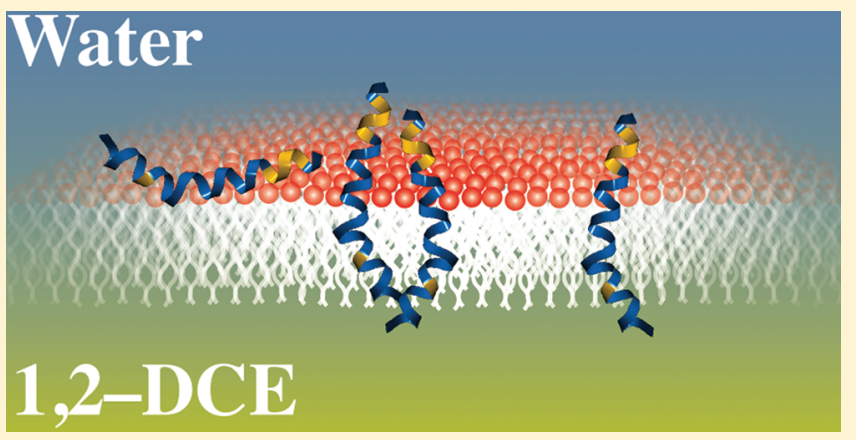
adsorption occurs near the ion transfer potential. Finally, the interaction of melittin with a monolayer of L- $\alpha$-dipalmitoyl phosphatidylcholine (DPPC) was also investigated showing that melittin destabilizes the lipidic monolayer facilitating its desorption. The non-covalent complex formation between melittin and DPPC was confirmed by mass spectrometry.

\section{INTRODUCTION}

Antimicrobial peptides are of crucial interest given their wide presence in nature. They constitute a powerful defense system against external potentially harmful agents such as bacteria and viruses. The challenge of bacterial resistance to conventional antibiotics, ${ }^{1}$ the antibacterial selectivity of many peptides, and their unique mode of action, have made such peptides promising candidates for the development of a new class of antibiotics. However, additional efforts are required for rationalizing their molecular interactions with lipidic membranes and their action mechanisms.

Among the antimicrobial peptide, melittin is certainly one of the most widely studied. This peptide is the principal toxic component of European honey bee venom ${ }^{3}$ and is a cationic hemolytic peptide composed of 26 amino acid residues whose amphiphilic nature makes it capable of associating with either natural or artificial lipid membranes. ${ }^{4}$ This pore-forming ability ${ }^{5}$ turns it into a suitable model peptide for monitoring lipidprotein interactions. Some of the analytical approaches used up to now to monitor this type of system include fluorescence, ${ }^{6}$ circular dichroism, ${ }^{7}$ surface plasmon resonance (SPR), ${ }^{8}$ nuclear magnetic resonance (NMR), ${ }^{9}$ and electrochemical impedance spectroscopy. ${ }^{10}$

However, probing interfacial interactions at cell membranes is not always an easy task given their complexity. Therefore, in the search of simpler but still realistic systems able to mimic the properties of cellular membranes, vesicle suspensions and immobilized bilayers or monolayers have been proposed. Nonetheless, the potential difference across the layers cannot be controlled or is neglected in many cases. Another interesting approach is based on adsorbed monolayers at polarized liquidliquid interfaces, where the control of the interfacial polarization is an important experimental variable and is accurately controlled. Adsorbed phospholipid monolayers at the ITIES were pioneered by Koryta et al. ${ }^{11}$ followed by interfacial tension measurements ${ }^{12}$ that clearly demonstrated the $\mathrm{pH}$ and potential dependence of these adsorption processes.

Since then, phospholipid monolayers adsorbed at liquidliquid interfaces formed between two immiscible electrolyte solutions (ITIES) have been used to mimic the behavior of lipid bio-membranes, ${ }^{12}$ especially to investigate the stability ${ }^{13}$ and ion permeability ${ }^{14}$ of the monolayers as a function of the well-defined interfacial potential difference. In parallel, less than two decades ago, the use of aqueous (organic) droplets supported on an electrode and immersed into an organic (aqueous) electrolyte solution to study electron transfer reactions coupled to ion transfer reactions was proposed. ${ }^{15}$ In these three-phase junctions (metal-water-oil), one of the interfaces is made not polarizable

Received: July 29, 2011

Revised: September 21, 2011

Published: September 30, 2011 
to study charge transfer reactions at the other interface. For example, for an aqueous droplet on a platinum electrode immersed in an organic electrolyte solution, the presence of an aqueous redox couple in excess in the droplet fixes the potential difference at the metal-water interface according to the Nernst equation for this couple, while the liquid-liquid (water-oil) interface is polarizable. Thus, a common three-electrode electrochemical setup can be used to study charge transfer reactions at the interface between two immiscible electrolyte solutions (ITIES). ${ }^{15 a}$ Additionally, the volume of one of the two phases can be dramatically reduced using this system. The latter is a key advantage for the study of biologically active molecules, which are very often available in very limited amounts.

Besides, the three-phase junction geometry has received also a lot of attention not only for studying ion transfer reactions ${ }^{16}$ but also for electro-wetting applications. ${ }^{17}$ In particular, Monroe et al. have considered the case in which ion-impermeable liquid-liquid interfaces are polarized ${ }^{18}$ to vary the contact angle of the droplet and have shown that relatively small applied voltages can induce very large drop shape variation. With video image digitizers, the shape of the droplet can be analyzed to estimate changes in the interfacial tension, and the three-phase junctions provide an easy way to accomplish this for the polarized ITIES. $^{12,19}$

Herein, we study the interaction between melittin with L- $\alpha$ dipalmitoylphosphatidylcholine (DPPC) monolayers adsorbed at the ITIES under controlled interfacial polarization using a three-phase junction in which the amount of peptide required is greatly reduced. Additionally, real-time videos were recorded during the electrochemical measurements to monitor the contact angle along with voltammetric signals to corroborate not only the adsorption of the peptide but also the potential region where it takes place.

Finally, to prove the existence of the complex formation between melittin and DPPC at the water-DCE interface, biphasic electrospray ionization (BESI) ${ }^{20}$ mass spectrometry was employed.

\section{EXPERIMENTAL SECTION}

Chemicals. Melittin 95\% (Mel) was purchased from Serva electrophoresis. The purity of the peptide was confirmed by UV visible, fluorescence, and mass spectrometry and was used without any further purification. L- $\alpha$-Dodecylphosphatydilcholine (DPPC) $(M=734.0 \mathrm{~g} /$ $\mathrm{mol}$ ) and acetic acid $(\mathrm{AcOH})$ were from Sigma (St. Louis, MO). $\mathrm{FeSO}_{4}$ and $\mathrm{Fe}_{2}\left(\mathrm{SO}_{4}\right)_{3}$ salts were from Merck. DCE was purchased from AppliChem (GmbH, Germany), and deionized water (18.2 $\mathrm{M} \Omega \mathrm{cm}^{-1}$ ) was prepared using a Milli-Q system from Millipore (Bedford, MA). For the electrochemical measurements, the organic salt used was bis(triphenylphosphoranylidene) ammonium tetrakis(pentafluorophenyl) borate $\left(\mathrm{BA}^{+} \mathrm{TB}^{-}\right)$. This salt was obtained by metathesis of bis(triphenylphosphoranylidene) ammonium chloride with lithium tetrakis(pentafluorophenyl)borate (all bought from Fluka). Purification was accomplished by recrystallization in acetone. All of the other employed compounds were of analytical grade and used as received, unless otherwise stated.

Electrochemical Measurements. Voltammetric measurements were carried out using a potentiostat (Autolab-PGSTAT 30, Metrohm) and a glass cubic cell. The cell was first cleaned by chrom-sulfuric acid and rinsed thoroughly with ultrapure water. The $i R$ potential drop was compensated using a positive-feedback method. A Pt disk electrode (Metrohm CH, Area $=0.06 \pm 0.01 \mathrm{~cm}^{2}$ ) was freshly polished sequentially with $1.0,0.3$, and $0.05 \mu \mathrm{m}$ alumina and sonicated for $5 \mathrm{~min}$ in
Scheme 1. Electrochemical Cell Employed

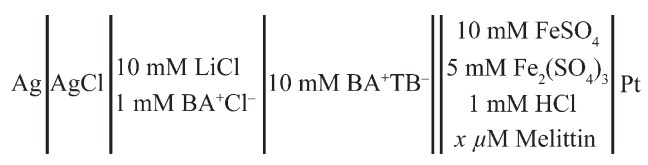

Scheme 2. Illustration of an Aqueous Droplet Supported on a Platinum Electrode Immersed in DCE

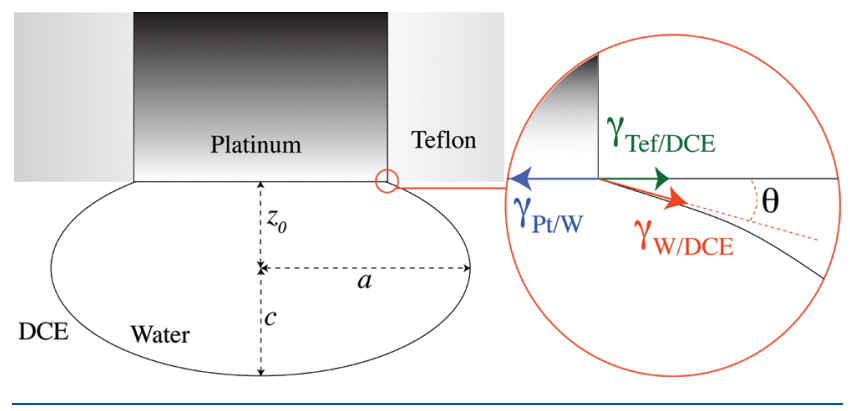

water/acetonitrile (50/50) mixture. A $10 \mu \mathrm{L}$ drop of an aqueous solution containing an equimolar mixture of $\mathrm{Fe}^{2+} / \mathrm{Fe}^{3+}$ ion pair was deposited using a micropipet onto the platinum disk electrode. The electrochemical cell composition can be represented as shown in Scheme 1, where $x$ stands for the concentration of peptide. All of the potentials for ion transfer are referred to the half-wave potential of tetraethylammonium $\left(\mathrm{TEA}^{+}\right)$. Thus, at the end of each experiment, a predetermined amount of a solution in 1,2-DCE of $\mathrm{TEA}^{+} \mathrm{TB}^{-}$is added to the organic phase in the cell for determining the experimental TEA ${ }^{+}$ half-wave potential. The value of the standard transfer potential of TEA ${ }^{+}$ has been taken equal to $18 \mathrm{mV} .{ }^{21}$ All of the measurements were carried out at room temperature of $22 \pm 1{ }^{\circ} \mathrm{C}$. The $\mathrm{pH}$ of the aqueous solution was maintained at a constant value of 3 , ensuring the stability of the redox pair in the aqueous solution as well as that of the peptide. Parallel experiments demonstrated that equivalent results could be obtained at either $\mathrm{pH} 3$ or $\mathrm{pH}$ 7. This is in accordance with the predicted titration curve of melittin (Figure S1), which reveals that the charge remains constant within a $\mathrm{pH}$ interval comprehended between approximately 2.5 and 8 .

Contact Angle Determination. Videos of the droplet during the voltammetric measurement were obtained with a hand-held microscope (Proscope HR, Bodelyn Technologies, U.S.). Video manipulation was carried out with Mathematica 7.0 (Wolfram Research) to calculate the contact angle at the four-phase boundary.

For analyzing the images, oblate spheroid geometry was assumed for the droplet. The general equation representing it corresponds to:

$$
\frac{x^{2}+y^{2}}{a^{2}}+\frac{z^{2}}{c^{2}}=1
$$

where $a$ is the equatorial radius and $c$ is the vertical radius. At $y=0$, the equation for the spheroid can be reduced to that of a simple ellipse, and therefore the area and the volume of the truncated spheroid can be calculated as follows:

$$
\begin{aligned}
& A=2 \pi \int_{-c}^{z_{0}} g(z) \sqrt{1+\frac{\mathrm{d}^{2} g(z)}{\mathrm{d} z^{2}}} \mathrm{~d} z \\
& V=\pi \int_{-c}^{z_{0}} g(z) \mathrm{d} z
\end{aligned}
$$


where $z_{0}$ is the vertical distance from the center at which the spheroid is truncated (see Scheme 2). The function $g(z)$ reads:

$$
g(z)=a \sqrt{1-\left(\frac{z}{c}\right)^{2}}
$$

Moreover, if one considers that the platinum and Teflon surfaces remain virtually unchanged upon polarization, the force balance at the boundary region $\mathrm{Pt}-\mathrm{Teflon}-$ water-DCE is related to the contact angle, $\theta$, as follows:

$$
\gamma_{\mathrm{Pt} / \mathrm{w}}=\gamma_{\mathrm{Tef} / \mathrm{DCE}}+\gamma_{\mathrm{w} / \mathrm{DCE}} \cos \theta
$$

which can also be expressed as:

$$
\sec (\theta)=\frac{\gamma_{\mathrm{w} / \mathrm{DCE}}}{\gamma_{\mathrm{Pt} / \mathrm{w}}-\gamma_{\mathrm{Tef} / \mathrm{DCE}}} \approx \frac{\gamma_{\mathrm{w} / \mathrm{DCE}}}{k}
$$

where the difference between $\gamma_{\mathrm{Pt} / \mathrm{w}}$ and $\gamma_{\mathrm{Tef} / \mathrm{DCE}}$ is assumed to remain nearly constant at a value $k$, which is in principle independent of the externally applied potential during the time scale of voltammetric measurements. Therefore, the interfacial tension between water and DCE is inversely proportional to the cosine of the contact angle. However, it must be stressed that this approach is not a methodology for measuring interfacial tension. Even if $\gamma_{\mathrm{Pt} / \mathrm{w}}$ and $\gamma_{\mathrm{Tef} / \mathrm{DCE}}$ remain constant in the course of a single experiment, their values might drastically change if the composition of the cell is modified, for example, when adding different species or when passing large currents across the interface. Yet, this approach will be shown to be of great value to monitor adsorption/desorption processes during linear scan voltammetry. As a result, if $a, c$, and $z_{0}$ are graphically determined from video snapshots taken during a voltammetric measurement, we can measure the contact angle using:

$$
\sec (\theta)=-\sec \left(\arctan \left(\left.\frac{\mathrm{d} g(z)}{\mathrm{d} z}\right|_{z=z_{0}}\right)\right)=\sqrt{1+\frac{a^{2} z_{0}^{2}}{c^{2}\left(c^{2}-z_{0}^{2}\right)}}
$$

For the image analysis, all of the snapshots are converted to grayscale, and the droplet contour is determined using a program written in Mathematica 7.0. Finally, the fitting quality can also be evaluated if one takes into account the following constraints:

(1) The volume of the droplet is constant; that is to say, it is independent of the potential.

(2) The transversal area of the droplet in contact with the electrode is constant and corresponds to the area of the platinum electrode given that the aqueous phase volume was always large enough as to ensure the complete coverage of the metallic surface.

MS Setup and Microspray Interface. An LTQVELOS ion trap mass spectrometer (Thermo Fisher Scientific, San Jose, CA) was used in positive ionization mode. The inlet capillary was kept at $100{ }^{\circ} \mathrm{C}$. The commercial electrospray interface was removed and replaced by a microspray interface consisting of a double microchannel $(20 \mu \mathrm{m} \times$ $50 \mu \mathrm{m} \times 1 \mathrm{~cm}$ ) polyimide microchip developed by DiagnoSwiss S.A. (Monthey, Switzerland) and fixed in a holder mounted on the probe slide adapter of the mass spectrometer. Further details have been previously described. ${ }^{20 \mathrm{~b}}$ The syringe pump (KdScientific, Holliston, MA) was used to provide constant flow rate of each solution through the microchannels $(2 \mu \mathrm{L} / \mathrm{min}$ for each line, i.e., a total flow rate of $4 \mu \mathrm{L}$ / $\mathrm{min})$. Two immiscible liquids are infused and put in contact only at the Taylor cone. The high voltage $(U=3.5 \mathrm{kV})$ was applied to the stainlesssteel needle of the syringe containing the aqueous solution. The current of spray was set between 30 and $80 \mathrm{nA}$ by adjusting the distance between the microspray outlet and the entrance of the MS and was monitored by a nanoammeter.

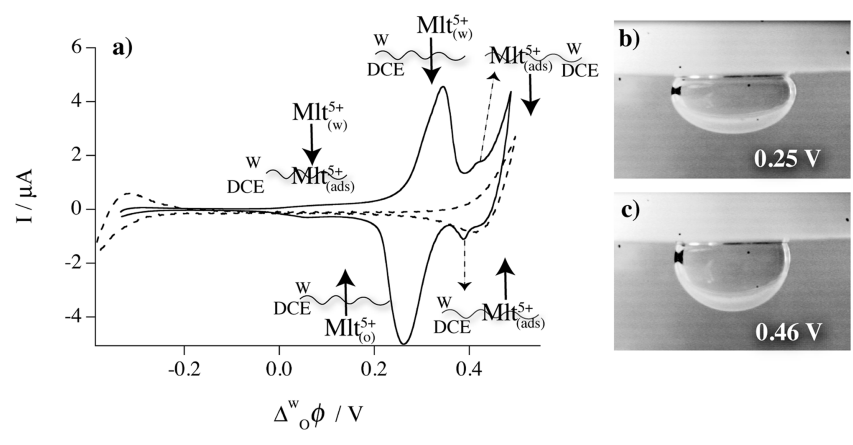

Figure 1. (a) Cyclic voltammogram obtained at the interface formed between DCE containing $\mathrm{BA}^{+} \mathrm{TB}^{-} 10 \mathrm{mM}$ and an aqueous droplet supported on a Pt disk electrode in the absence (dashed line) and in the presence (solid line) of melittin $80 \mu \mathrm{M}$. Scan rate $=10 \mathrm{mV} \mathrm{s}^{-1}$. In (b) and (c), video snapshots at different galvanic potentials of an aqueous droplet containing melittin $(x=20 \mu \mathrm{M})$ and DCE in the absence of DPPC are presented.

\section{RESULTS AND DISCUSSION}

Voltammetric Behavior of Melittin. Figure 1a shows a cyclic ion transfer voltammogram of an aqueous droplet containing melittin $80 \mu \mathrm{M}$. Despite the asymmetric distribution of polar and nonpolar residues and the fact that like other membrane-binding peptides and proteins, melittin is predominantly hydrophobic and adopts an $\alpha$-helical conformation (see Figure S1), ${ }^{22,23}$ a clear ion transfer process for melittin can be identified. At the same time, evident deformation of the aqueous droplet could also be observed at potentials close to the ion transfer potential (Figure 1b). The half-wave potential corresponds to $0.28 \mathrm{~V}$, that is, a free Gibbs energy of transfer of $135 \mathrm{~kJ} \mathrm{~mol}^{-1}$. The peak-topeak separation extrapolated to zero scan rate was determined to be $17 \mathrm{mV}$, which differs from the $12 \mathrm{mV}$ peak separation expected for an ion carrying five positive charges, according to the calculated charge versus $\mathrm{pH}$ profile in Figure S1.

Nonetheless, it has to be stressed that accurate peak separation determination from cyclic voltammetry data can be very difficult for heavily charged species. In fact, the accuracy with which the peak separation potential is limited to ca. $5 \mathrm{mV}$. In addition to this, the charge of the peptide might differ from that theoretically predicted because of ion-pair formation with ions of the supporting electrolyte or within the same molecule. ${ }^{24}$ Indeed, intramolecular ion-pairing could take place at the interface or in the organic phase between the two opposite charges K-20 and G-26.

Taking this into account, the ion transfer signal for melittin can be categorized as quasi-reversible, in accordance with the size of this peptide and the lowering effect that this parameter plays on the ion transfer kinetic constant. ${ }^{25}$

Further experiments in the presence of the peptide showed small variations of the peak potential upon variation of both scan rate and concentration (Figure 2). However, linear relationships are always obtained between the peak currents and the square root of the scan rate (inset Figure $2 \mathrm{a}$ ) and the concentration (inset Figure $2 \mathrm{~b}$ ). Additionally, a small signal, but significant enough to be seen, located at approximately $0.4 \mathrm{~V}$ is observed (Figures 1a and 2). This signal is present in both forward and backward scans and seems to be related to the adsorption process of melittin at the water-DCE interface. Nonetheless, given the low magnitude of this peak as well as its close proximity to the melittin diffusion-controlled wave and the end of the potential 

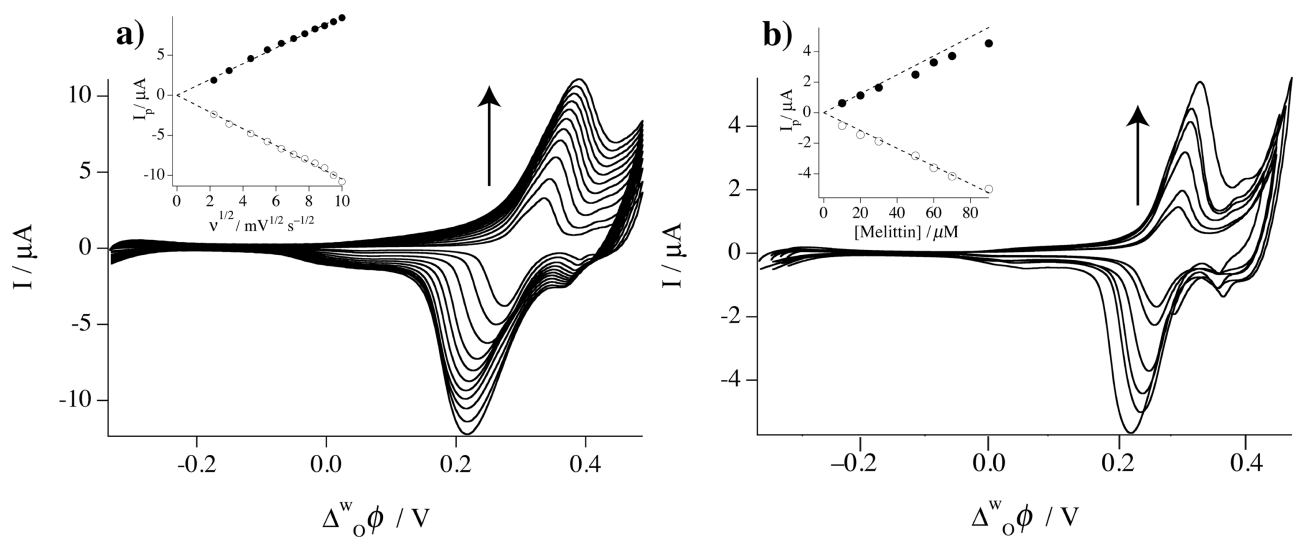

Figure 2. (a) Ion-transfer voltammograms obtained at the interface formed between DCE in the absence of DPPC and an aqueous solution of (a) melittin $(x=80 \mu \mathrm{M})$ and DCE in the absence of DPPC at scan rates of 5, 10, 20, 30, 40, 50, 60, 70, 80, 90, and $100 \mathrm{mV} \mathrm{s}^{-1}$. In (b), the scan rate was kept constant at $10 \mathrm{mV} \mathrm{s}^{-1}$ and for $x=20,30,50,60,80$, and $90 \mu \mathrm{M}$. The dependencies of the peak signals on the scan rate and concentration are presented in the insets of (a) and (b), respectively.

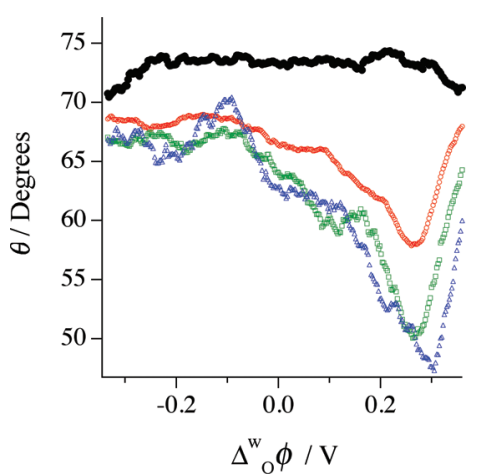

Figure 3. Four-phase junction boundary angle dependence on the galvanic potential across the interface formed between DCE in the absence of DPPC and an aqueous solution containing melittin at concentrations of (black) 0, (red) 20, (green) 50, and (blue) $100 \mu \mathrm{M}$ at a scan rate of $10 \mathrm{mV} \mathrm{s}^{-1}$.

window, it is then difficult to judge about the real nature of this peak by relying only on voltammetric data.

It can be then concluded that the ion-transfer of melittin corresponds to a quasi-reversible and diffusion-controlled process slightly distorted because of the adsorption of the aqueous peptide. Nonetheless, to better describe the adsorption process, real-time video snapshots were acquired simultaneously with the cyclic voltammogram (see Figure S2). As mentioned above, deformation of the shape of aqueous droplet was observed during the voltammetric measurements. This phenomenon can be rationalized in terms of an adsorption process in which the energy per unit of area will be reduced. Accordingly, this induces an increase in the area of the drop given that the volume of the droplet is fixed. Equally, the angle $\theta$ at the four-phase junction shown in Scheme 2 is considerably modified. Video analysis was used to monitor this angle variation, and the results are plotted in Figure 3. At negative potentials, the droplet is rather spherical, and the surface tension is high. When scanning to positive potentials, the contact angle value decreases as the aqueous droplet becomes gradually flatter, leading to a minimum in the contact angle profile as shown in Figure 3, which is associated with the adsorption of melittin. This minimum lies close to the standard ion transfer potential (herein assumed to be approximately equal to the half-wave potential). ${ }^{26}$ Upon further scanning to positive potentials, melittin desorbs and the drop recovers its quasi-spherical shape. Increasing the concentration of the peptide was also observed to have a marked influence on this process; however, the present video technique is too coarse to measure the adsorption isotherm. Nevertheless, it can be seen that at higher concentrations the angle tends to be reduced to a larger extent. However, if the concentration is raised beyond a certain threshold (ca. $100 \mu \mathrm{M}$ ), the variation of the angle minimum becomes independent of the concentration (data not shown), meaning that saturation of the liquid-liquid interface with the peptide takes place above this concentration value. Additionally, video analysis of the forward and backward scans showed that the adsorption process can be assumed as reversible in the time scale of voltammetric experiments. Indeed, angle versus potential curves presented in Figure S3 do not present hysteresis upon reversing the scan direction. Additionally, repeated cycles did not show any sign of modification of the interface. It must be stressed that very commonly ionic adsorption at liquid-liquid interfaces is accompanied by the formation of aggregates or ion-pairs, which modify in an irreversible manner the interfacial region. ${ }^{27}$ In stark contrast, melittin, besides being able to be transferred across the interface, also adsorbs in a reversible manner at the interface.

By analogy with voltammetry for redox reactions at solid electrodes where the oxidized or reduced species can be adsorbed and where post-peaks correspond to the adsorption of the reactant of the redox reaction and pre-peaks correspond to the adsorption of the product of this reaction, post-peaks (prepeaks) in the ion transfer voltammogram correspond to the adsorption of the transferring (transferred) ions as recently discussed. ${ }^{26 \mathrm{~b}}$ Clear evidence of ion transfer accompanied by adsorption of the transferring ion has also been previously documented. ${ }^{28}$ However, no clear experimental confirmation for the existence of well-defined adsorptive pre- or post-peaks has been reported so far for polypeptides and in particular for antimicrobial peptides.

In Figure 1, the post-peak on the scan to positive potentials can be attributed to the transfer by desorption of adsorbed $\mathrm{Mel}_{(\mathrm{ads})}$ that requires more energy to transfer than the bulk species $\mathrm{Mel}_{(\mathrm{w})}$, according to the $\theta$ profile as a function of potential in Figure 3. Thus, combining the contact angle measurements with 


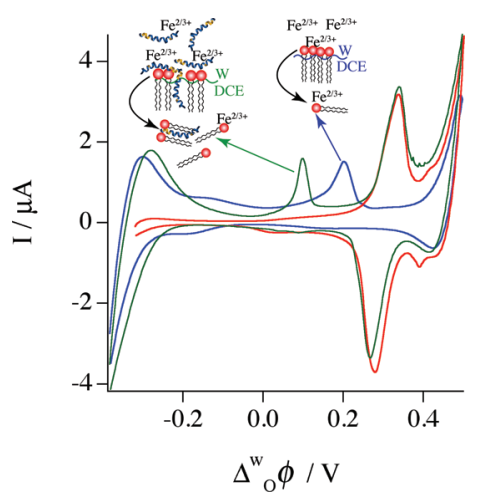

Figure 4. Ion-transfer voltammograms obtained at the interface formed between DCE in the absence (red) and in the presence (blue and green) of DPPC and an aqueous solution in the absence (blue) and in the presence (red and green) of melittin $80 \mu \mathrm{M}$. Scan rate $=10 \mathrm{mV} \mathrm{s}^{-1}$.

the voltammetry data, it is possible to describe the phenomena taking place when scanning to positive potentials while using a very small amount of the peptide of interest. At negative potential values, the adsorption of the peptide does not take place in an appreciable extent, as seen in Figure 3. Indeed, upon scanning, aqueous melittin only starts to adsorb at about $-0.1 \mathrm{~V}$. In fact, given that melittin is a charged species, its adsorption process is potential dependent; ${ }^{29}$ that is, the more positive is the applied potential, the higher is the surface coverage, the lower is the interfacial tension, and therefore the lower is the contact angle. At about $0.28 \mathrm{~V}$, melittin coming from the aqueous bulk transfers across the interface, and the adsorbed layer then starts to desorb toward the organic phase. This desorption process, according to Figure 3, is completed at ca. $0.4 \mathrm{~V}$, causing the appearance of the post-peak aforementioned.

When the scan direction is reversed, a pre-peak corresponding to a transfer facilitated by adsorption of melittin followed by the ion transfer wave from the melittin returning to the aqueous phase is observed. It is remarkable that this antimicrobial peptide provides such a clear voltammetric response, and this is to the best of the authors' knowledge the first report of a quasireversible adsorption-transfer of an antimicrobial peptide at the ITIES.

Melittin-DPPC System. Because melittin is a well-known antimicrobial peptide capable of disrupting into the cell membrane, its effect on lipidic monolayers at the water-1,2-DCE interface was also investigated. As shown in Figure 5, when only DPPC is present in the organic phase, dramatic changes are observed in the contact angle. This is in agreement with previous reports in which strong adsorption of phosphatidylcholine has been observed at the ITIES. ${ }^{12,13 d, 30}$ Additionally, the appearance of a desorptive signal in the absence of peptide can be observed at approximately $0.2 \mathrm{~V}$, which stems from the complexation process of either $\mathrm{Fe}^{2+}$ or $\mathrm{Fe}^{3+}$ with the zwitterionic phospholipids at the liquid-liquid interface and further desorption from the ITIES to the organic phase (Figure 4). This gives rise to a positive current in the forward scan because aqueous cations are being ultimately transferred to the DCE phase. Similar behavior has been identified for cations such as protons, ${ }^{31}$ calcium, ${ }^{32}$ potassium, ${ }^{13 \mathrm{~d}, 33}$ etc.

When melittin is present in the aqueous phase, the position of this desorptive peak is shifted toward less positive potentials, while the transfer of the peptide remains unaltered as can be seen in Figure 4 (red line). This indicates that desorption of the

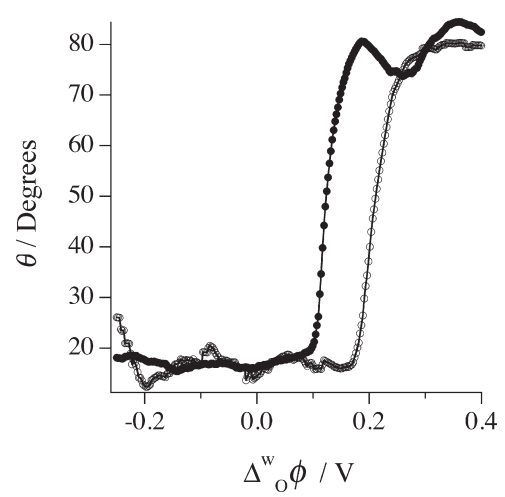

Figure 5. Four-phase junction boundary angle dependence on the galvanic potential across the interface formed between DCE containing DPPC $30 \mu \mathrm{M}$ and an aqueous droplet in the absence $(O)$ and in the presence $(O)$ of melittin $(x=20 \mu \mathrm{M})$ obtained for the forward scan at $10 \mathrm{mV} \mathrm{s}^{-1}$.

phospholipid from the interface occurs earlier in the presence of the peptide, as depicted in the inset of the same figure. These results are very different from those previously reported for a smaller, non surface-active positively charged peptide like Angiotensin III: ${ }^{20 a}$

(1) For Angiotensin III, a diminution in its ion transfer potential was observed in the presence of DPPC. Therefore, the phospholipid was recognized to be an ionophoric layer that facilitates the peptide transfer reaction rather than a barrier.

(2) The phospholipid desorption occurs easily (i.e., at less positive potentials) in the presence of melittin.

Further confirmation of these experimental facts was made possible by the contact angle measurements shown in Figure 5. It is clear from this figure that a strong adsorption of DPPC takes place at negative potentials and it starts desorbing form the interface at positive potentials. In the absence of melittin, such a desorption process starts at ca. $0.18 \mathrm{~V}$. This desorption process along with the complexation of aqueous cations is the responsible for current peak present at $0.2 \mathrm{~V} .{ }^{31}$ Nonetheless, in the presence of melittin, the onset of the desorption process is estimated from the contact angle measurements to be present at $0.1 \mathrm{~V}$. As discussed above, melittin starts to adsorb at $0.1 \mathrm{~V}$. These data therefore suggest that upon adsorption melittin penetrates into the phospholipid monolayer, thereby destabilizing it and catalyzing its desorption from the interface.

Thus, melittin facilitates the lipid desorption process where the phospholipid can be seen as an ionophore for this highly charged peptide to transfer into the organic phase. In fact, because of the big size of the complex formed between melittin peptide and DPPC phospholipid as compared to its isolated precursors, this complex would be significantly more hydrophobic, and accordingly its transfer to the organic phase would require less energy input. Additionally, once the lipid is displaced from the interface, the transfer of aqueous melittin occurs at the same potentials as observed previously in the absence of DPPC (Figure 3). This indicates that the lipid monolayer has been entirely removed at potentials close to the standard ion transfer potential of melittin.

Mass Spectrometric Analysis. Finally and to confirm the existence of a non-covalent complex between melittin and DPPC, we used the technique of biphasic electrospray ionization mass spectrometry (BESI-MS) in which the microchip emitter is able to form a liquid-liquid interface within the Taylor cone, 


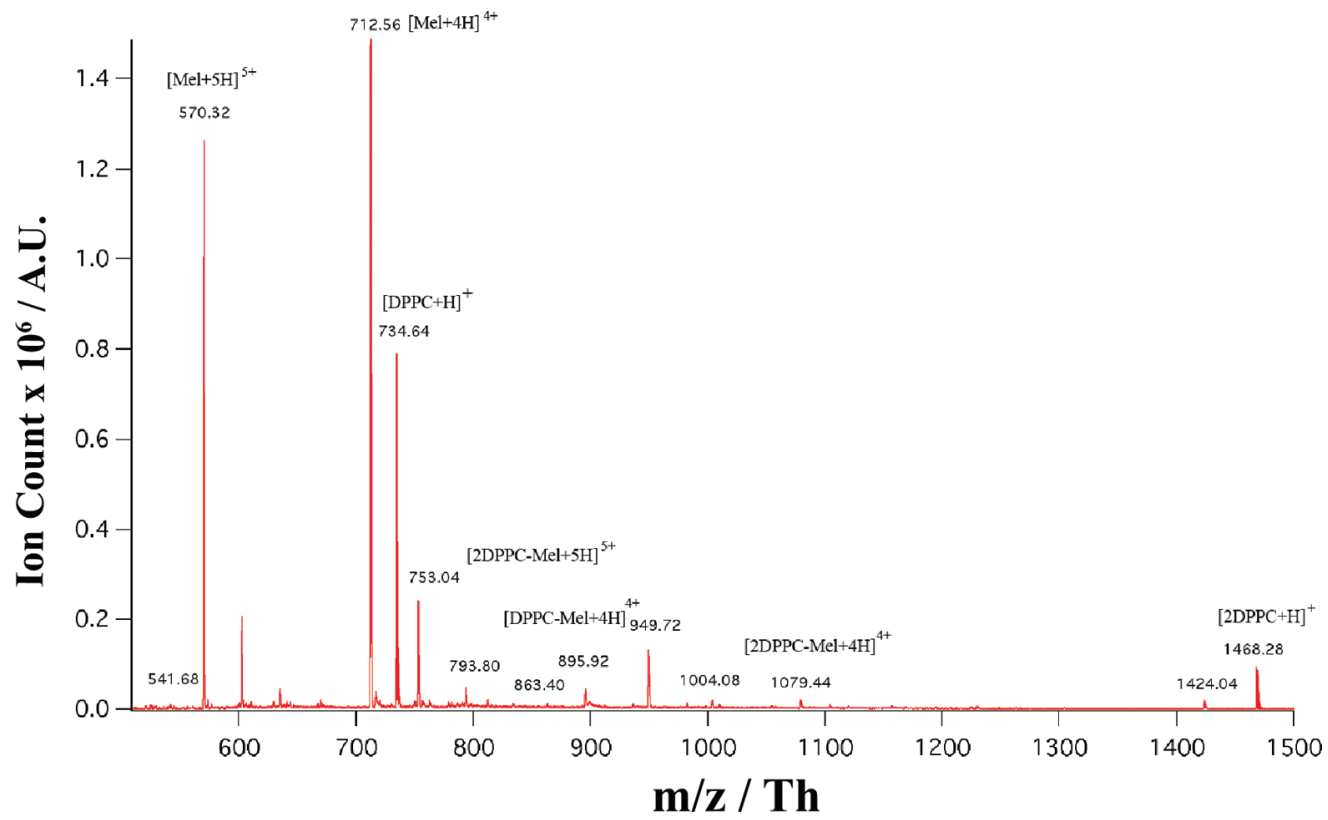

Figure 6. Mass spectrum of the reaction between DPPC $200 \mu \mathrm{M}$ in DCE and melittin $50 \mu \mathrm{M}$ in acidified water (1\% acetic acid) with a BESI source coupled to the time-of-flight mass spectrometer.

where emulsification induced by the electric field is likely to take place. In this configuration, one channel is dedicated to the organic solvent and was filled with $200 \mu \mathrm{M}$ DPPC in pure 1,2$\mathrm{DCE}$, and the other line was filled with acidified water $\mathrm{AcOH}$ at $1 \%)$ containing $50 \mu \mathrm{M}$ melittin. The results are presented in Figure 6. DPPC and its dimer were observed at $\mathrm{m} / z=$ 734.6 Th and $m / z=1468.28$ corresponding to $[\mathrm{DPPC}+\mathrm{H}]^{+}$and $[2 \mathrm{DPPC}+\mathrm{H}]^{+}$, respectively. Moreover, the ions of $[\mathrm{Mel}+4 \mathrm{H}]^{4+}$ and $[\mathrm{Mel}+5 \mathrm{H}]^{5+}$ were observed at $\mathrm{m} / z$ values of 712.56 and $570.32 \mathrm{Th}$, respectively. The complex formation between DPPC and Melittin, marked as $[\mathrm{DPPC}-\mathrm{Mel}+4 \mathrm{H}]^{4+}$ in the spectrum, was also confirmed, and its corresponding signals can be appreciated at $\mathrm{m} / z$ values of $895.9 \mathrm{Th}$. Additionally, the DPPC dimer $\left([2 \mathrm{DPPC}+\mathrm{H}]^{+}\right)$, as well as its complexes with melittin ([2DPPC-Melittin $+4 \mathrm{H}]^{4+}$ and $\left.[2 \mathrm{DPPC}-\text { Melittin }+5 \mathrm{H}]^{5+}\right)$, are observed at $m / z=1468.28 \mathrm{Th}$, 1079.44 Th, and 863.40 Th, respectively. This type of complexation reaction in which multiple phospholipid molecules are involved could constitute the initial steps of an early stage of the reverse micelles formation process, as was previously reported as responsible for protein extraction in organic solvents. Similar results have been observed for the complex formation between peptides like angiotensin III and leucine-enkephalin with DPPC. ${ }^{20 a}$

\section{CONCLUSION}

The adsorption of melittin at the liquid-liquid interface has been demonstrated by voltammetry and confirmed by real-time image analysis of video snapshots. More importantly, we have shown that this highly charged antimicrobial peptide provides very clear ion transfer voltammetry data while using a very small amount of it. Additionally, the present methodology made possible the acquisition of contact-angle profiles at the fourphase junction. These measurements indicated that the maximum surface coverage is attained in the proximity of the standard ion transfer potential. Furthermore, the interaction of this cytolytic peptide with a model phospholipid monolayer was proved to induce the desorption of the lipid at less positive potentials from the interface. As a matter of fact, comparison of contact angle versus potential across the interface revealed that the onset of this desorptive process is located in the potential region where the antimicrobial peptide adsorption begins. In light of these results, we propose that the membrane-disruption ability of this peptide is intimately linked to its direct interaction with the lipid upon potential dependent adsorption. Preliminary comparison of this work with previous reports ${ }^{20 \mathrm{a}}$ also indicates that voltammetry at ITIES combined with contact angle measurements is a suitable technique for future studies in which clear distinction between cytolytic and noncytolytic peptides could be achieved.

These results besides confirming predicted voltammetric signals stemming from adsorption processes set the basis of a new methodology for the study of antimicrobial peptides as a function of the potential difference across the interface. The existence of noncovalent complexes between the melittin peptide and DPPC lipid was equally confirmed by mass spectrometry.

Finally, the significant changes in the contact angle within a small potential region open the way for the study and potential applications of this system in the conception of new electrovariable lenses, micro-fluidic devices, etc.

\section{ASSOCIATED CONTENT}

S Supporting Information. Calculated charge for melittin as a function of $\mathrm{pH}$. Detailed snapshot sequence of the forward scan in the presence of melittin and determined contact angles for the forward and backward scans. This material is available free of charge via the Internet at http://pubs.acs.org.

\section{AUTHOR INFORMATION}

\section{Corresponding Author}

*Fax: +41 21693 3667. E-mail: hubert.girault@epfl.ch.

\section{Author Contributions}

"These authors contributed equally to this work. 


\section{ACKNOWLEDGMENT}

The Fonds National Suisse pour la Recherche Scientifique is thanked for financial support through the project "Electrochemical methodology for the study of peptide lipid interaction" (Grant no. 200020-113428). Technical assistance by Valérie Devaud is also acknowledged.

\section{REFERENCES}

(1) Davies, J. Nature 1996, 383, 219-220.

(2) Hancock, R.; Lehrer, R. Trends Biotechnol. 1998, 16, 82-88.

(3) Haberman, E. Science 1972, 177, 314-322.

(4) (a) Faucon, J. F.; Dufourcq, J.; Lussan, C. FEBS Lett. 1979, 102, 187-190. (b) Bernheimer, A. W.; Rudy, B. Biochim. Biophys. Acta 1986, 864, 123-141. (c) Dempsey, C. E. Biochim. Biophys. Acta 1990, $1031,143-161$.

(5) (a) Saberwal, G.; Nagaraj, R. Biochim. Biophys. Acta 1994, 1197, 109-131. (b) Matsuzaki, K.; Yoneyama, S.; Miyajima, K. Biophys. J. 1997, 73, 831-838.

(6) Raghuraman, H.; Chattopadhyay, A. Biophys. J. 2004, 87, 24192432.

(7) Beschiaschvili, G.; Seelig, J. Biochemistry 1990, 29, 52-58.

(8) (a) Mathur, S.; Badertscher, M.; Scott, M.; Zenobi, R. Phys. Chem. Chem. Phys. 2007, 9, 6187-6198. (b) Ha, M. H.; Endo, T.; Saito, M.; Chikae, M.; Do, K. K.; Yamamura, S.; Takamura, Y.; Tamiya, E. Anal. Chem. 2008, 80, 1859-1864.

(9) Kuchinka, E.; Seelig, J. Biochemistry 1989, 28, 4216-4221.

(10) Becucci, L.; Leon, R. R.; Moncelli, M. R.; Rovero, P.; Guidelli, R. Langmuir 2006, 22, 6644-6650.

(11) Koryta, J.; Hung, L. Q.; Hofmanova, A. Stud. Biophys. 1982, 90, 25-29.

(12) Girault, H. H. J.; Schiffrin, D. J. J. Electroanal. Chem. 1984, $179,277-284$.

(13) (a) Santos, H. A.; Carlsson, S.; Murtomaki, L.; Kontturi, K. ChemPhysChem 2007, 8, 913-920. (b) Santos, H. A.; Ferreira, E. S.; Pereira, E. J.; Pereira, C. M.; Kontturi, K.; Silva, F. ChemPhysChem 2007, 8, 1540-1547. (c) Saint Martin, E.; Konovalov, O.; Daillant, J. Thin Solid Films 2007, 515, 5687-5690. (d) Yoshida, Y.; Maeda, K.; Shirai, O. J. Electroanal. Chem. 2005, 578, 17-24. (e) Kakiuchi, T.; Yamane, M.; Osakai, T.; Senda, M. Bull. Chem. Soc. Jpn. 1987, 60, 4223-4228.

(14) (a) Kakiuchi, T.; Kondo, T.; Kotani, M.; Senda, M. Langmuir 1992, 8, 169-175. (b) Huang, J.; Chen, L.; Zhang, X.; Liu, S.; Li, G. Electrochem. Commun. 2008, 10, 451-454. (c) Monzon, L.; Yudi, L. Electrochim. Acta 2006, 51, 4573-4581.

(15) (a) Ulmeanu, S.; Lee, H.; Fermin, D.; Girault, H.; Shao, Y. Electrochem. Commun. 2001, 3, 219-223. (b) Scholz, F.; Gulaboski, R.; Caban, K. Electrochem. Commun. 2003, 5, 929-934. (c) Scholz, F.; Gulaboski, R. ChemPhysChem 2005, 6, 16-28. (d) Liu, X. H.; Yang, J.; Zuo, G. F.; Zhang, K.; Dong, C. W.; Lu, X. Q. J. Phys. Chem. C 2008, $112,148-152$

(16) (a) Charreteur, K.; Quentel, F.; Elleouet, C.; L'Her, M. Anal. Chem. 2008, 80, 5065-5070. (b) Scholz, F.; Gulaboski, R.; Caban, K. Electrochem. Commun. 2003, 5, 929-934.

(17) Mugele, F.; Baret, J. C. J. Phys.: Condens. Matter 2005, 17, R705-R774.

(18) (a) Monroe, C. W.; Daikhin, L. I.; Urbakh, M.; Kornyshev, A. A. Phys. Rev. Lett. 2006, 97, 136102. (b) Monroe, C. W.; Daikhin, L. I.; Urbakh, M.; Kornyshev, A. A. J. Phys.: Condens. Matter 2006, 18, 2837-2869.

(19) Allen, R.; Kontturi, K.; Murtomaki, L.; Williams, D. J. Electroanal. Chem. 2000, 483, 57-67.

(20) (a) Méndez, M. A.; Prudent, M.; Su, B.; Girault, H. H. Anal. Chem. 2008, 80, 9499-9507. (b) Prudent, M.; Méndez, M. A.; Girault, H. H. Anal. Sci. 2008, 24, 1399-1404. (c) Prudent, M.; Mendez, M. A.; Jana, D. F.; Corminboeuf, C.; Girault, H. H. Metallomics 2010, 2, 400-406.

(21) Sabela, A.; Marecek, V.; Samec, Z.; Fuoco, R. Electrochim. Acta 1992, 37, 231-235.
(22) (a) Terwilliger, T. C.; Elisenberg, D. J. Biol. Chem. 1982, 257, 6010-6015. (b) Terwilliger, T. C.; Eisenberg, D. J. Biol. Chem. 1982, 257, 6016-6022.

(23) Humphrey, W.; Dalke, A.; Schulten, K. J. Mol. Graphics 1996, $14,33-38$

(24) Shinshi, M.; Sugihara, T.; Osakai, T.; Goto, M. Langmuir 2006, 22, 5937-5944.

(25) Kakiuchi, T.; Noguchi, J.; Senda, M. J. Electroanal. Chem. 1992, $327,63-71$.

(26) (a) Kakiuchi, T. J. Electroanal. Chem. 2001, 496, 137-142. (b) Méndez, M. A.; Su, B.; Girault, H. H. J. Electroanal. Chem. 2009, 634, 82-89.

(27) (a) Hartvig, R.; Méndez, M.; van der Weert, M.; Jorgensen, L.; Ostergaard, J.; Girault, H. H.; Jensen, H. Anal. Chem. 2010, 82, 7699-7705. (b) Herzog, G.; Kam, V.; Arrigan, D. W. M. Electrochim. Acta 2008, 53, 7204-7209.

(28) Goto, T.; Maeda, K.; Yoshida, Y. Langmuir 2005, 21, 1178811794 .

(29) Hartvig, R. A.; van de Weert, M.; Ostergaard, J.; Jorgensen, L.; Jensen, H. Langmuir 2011, 27, 2634-2643.

(30) Samec, Z.; Trojánek, A.; Krtil, P. Faraday Discuss. 2005, $129,301-313$.

(31) Janchenova, H.; Lhotsky, A.; Stulik, K.; Marecek, V. J. Electroanal. Chem. 2007, 601, 101-106.

(32) (a) Ohnishi, S.; Ito, T. Biochemistry 1974, 13, 881-887. (b) Chesniuk, S.; Dassie, S.; Yudi, L.; Baruzzi, A. Electrochim. Acta 1998, 43, 2175-2181.

(33) Monzón, L.; Yudi, L. Electrochim. Acta 2006, 51, 1932-1940. 